\title{
Totally laparoscopic central bisectionectomy for colorectal cancer liver metastasis using indocyanine green fluorescence imaging
}

\author{
Youngmok PARK*, Jaeri KIM, Hyungil SEO, Myunghee YOON
}

Department of Surgery, Pusan National University Hospital, Busan, Korea

Introduction: Laparoscopic central bisectionectomy is still an area of great challenge and technically requiring surgery due to high incidence of surgical complication and poor intraoperative outcomes. We report our experience totally laparoscopic central bisectionectomy for colorectal cancer liver metastasis (CRLM) using indocyanine green (ICG) fluorescence imaging.

Methods: The patient was 77-years-old man and was performed laparoscopic low anterior resection with ileostomy, cholecystectomy for rectal cancer 3 years before admission. He diagnosed metachronous CRLM and on magnetic resonance imaging, $5 \mathrm{~cm}$ sized hepatic mass was shown between segment 4 and 8 with middle hepatic vein (MHV) involvement. During operation, the Cavitron Ultra Sonic Aspirator ${ }^{\circledR}$ and Sonicision ${ }^{\mathrm{TM}}$ (Medtronic) was applied for hepatic parenchyma dissection. For resection of major glissonian pedicle and MHV, Signia ${ }^{\mathrm{TM}}$ staple (Medtronic) was used. After right anterior glissonian pedicle was isolated and temporary clamping, 2.5 mg ICG was injected intravenously. The ICG stained area could be recognized, and subsequent parenchyma dissection was completed successfully under Pringled maneuver. Specimen was retieved via extended umbilical port site.

Results: The operation time was 205 minutes and the estimated blood loss was $300 \mathrm{~mL}$. No transfusion was done intraoperatively and postoperatively. There was no significant complication after surgery. Computed tomography was checked on postoperative day 5 , showed no abnormal pathologic findings. On pathologic findings, metastatic adenocarcinoma sized $5.4 \mathrm{~cm}$ with clear resection margin was diagnosed. The patient discharged on postoperative day 7 .

Conclusions: Laparoscopic central bisectionectomy is safe and feasible compared with conventional open surgery. ICG imaging system is effective and helpful for safe hepatic resection. 